\title{
CONDITIONING PROPERTIES OF THE STATIONARY DISTRIBUTION FOR A MARKOV CHAIN*
}

\author{
STEVE KIRKLAND ${ }^{\dagger}$
}

\begin{abstract}
Let $T$ be an irreducible stochastic matrix with stationary vector $\pi^{T}$. The conditioning of $\pi^{T}$ under perturbation of $T$ is discussed by providing an attainable upper bound on the absolute value of the derivative of each entry in $\pi^{T}$ with respect to a given perturbation matrix. Connections are made with an existing condition number for $\pi^{T}$, and the results are applied to the class of Markov chains arising from a random walk on a tree.
\end{abstract}

Key words. Stochastic matrix, Stationary vector, Condition number.

AMS subject classifications. 15A51, 15A18, 65F35, 60J10.

1. Introduction. Suppose that $T$ is an $n \times n$ irreducible stochastic matrix; evidently we can think of such a $T$ as the transition matrix for a Markov chain. One of the central quantities of interest arising from $T$ is its stationary distribution, i.e. the positive vector $\pi^{T}$ satisfying $\pi^{T} T=\pi^{T}$, and normalized so that $\pi^{T} \mathbf{1}=1$, where 1 denotes the all ones vector.

A number of authors have considered the stability of $\pi^{T}$ under perturbation, and have addressed problems of the following type: if $T$ is perturbed to yield another irreducible stochastic matrix $\tilde{T} \equiv T+E$ with stationary vector $\tilde{\pi}^{T}$, can the quantity $\left\|\tilde{\pi}^{T}-\pi^{T}\right\|$ be bounded by $f(T)\|E\|$ (for some suitable pair of vector and matrix norms) where $f$ is some function of the matrix $T$. Such an $f(T)$ is known as a condition number for the Markov chain, and the survey of Cho and Meyer [3] collects and compares several condition numbers.

One condition number of interest discussed in that paper is defined as follows. Let $Q=I-T$, and form its group generalized inverse $Q^{\#}$ (see [2] for background on generalized inverses); now define $\kappa(T)$ by

$$
\kappa(T) \equiv \frac{1}{2} \max _{1 \leq j \leq n}\left\{Q_{j, j}^{\#}-\min _{1 \leq i \leq n} Q_{i, j}^{\#}\right\} .
$$

Denote the maximum absolute value norm of $\tilde{\pi}^{T}-\pi^{T}$ by $\left\|\tilde{\pi}^{T}-\pi^{T}\right\|_{\infty}$, and the maximum absolute row sum of $E$ by $\|E\|_{\infty}$; it is shown in [4] and [10] that

$$
\left\|\tilde{\pi}^{T}-\pi^{T}\right\|_{\infty} \leq \kappa(T)\|E\|_{\infty}
$$

Further, results in [3] and in [8] show that the condition number $\kappa(T)$ is the smallest among the eight condition numbers considered in [3]. In particular, if $\kappa(T)$ is small, then we can conclude that the stationary vector is stable under perturbation of $T$. (However, it turns out that $\kappa(T)$ cannot be too small; a result in [8] shows that for any irreducible transition matrix $T$ of order $n, \kappa(T) \geq \frac{n-1}{2 n}$.)

${ }^{*}$ Received by the editors on 23 April 2002. Final version accepted for publication on 14 January 2003. Handling editor: Michael Neumann.

${ }^{\dagger}$ Department of Mathematics and Statistics, University of Regina, Regina, Saskatchewan, Canada S4S 0A2 (kirkland@math.uregina.ca). Research supported in part by an NSERC Research Grant. 
On the other hand, if $\kappa(T)$ is large, then on the face of it, $\left\|\tilde{\pi}^{T}-\pi^{T}\right\|_{\infty}$ may still be small relative to $\|E\|_{\infty}$, so how accurate a measure of conditioning does $\kappa(T)$ provide? In this paper, we address that question by considering the derivative of $\pi^{T}$ with respect to a perturbation matrix $E$, and finding an attainable upper bound on the absolute value of that derivative. Among other consequences, we deduce that for each irreducible stochastic matrix $T$, there is a family of perturbation matrices with arbitrarily small norms, such that for each such matrix $E$, we have $\left\|\tilde{\pi}^{T}-\pi^{T}\right\|_{\infty}>$ $\frac{\kappa(T)\|E\|_{\infty}}{2}$. By way of illustrating our results, we close the paper by considering the transition matrices corresponding to random walks on trees. We characterize those trees so that the corresponding transition matrix $T$ has the property that for each perturbation matrix $E$ of sufficiently small norm, $\left\|\tilde{\pi}^{T}-\pi^{T}\right\|_{\infty}<\|E\|_{\infty}$.

Throughout $T$ will be our notation for an irreducible stochastic matrix of order $n$, and $\pi^{T}$ will denote its stationary vector. We define $Q$ by $Q \equiv I-T$, while $Q^{\#}$ will denote the group inverse of $Q$. We use $E$ to denote a perturbation matrix such that $\tilde{T}=T+E$ is also irreducible and stochastic, while $\tilde{\pi}^{T}$ will be our notation for the stationary vector for $\tilde{T}$. The class of admissible perturbation matrices will be denoted by $\mathcal{E}_{T}$, so that $\mathcal{E}_{T}=\{E \mid T+E$ is irreducible and stochastic $\}$. Observe that if $E \in \mathcal{E}_{T}$, then there are certain combinatorial constraints on the negative entries of $E$; for example, $E_{i, j}<0$ only if $T_{i, j}>0$. In particular, note that $-E$ may not be in $\mathcal{E}_{T}$. We will assume basic knowledge of graph theory, the theory of nonnegative matrices and the study of Markov chains; the reader is referred to [1] for background on the first topic, to [11] for background on the second, and to [6] for background on the last topic.

2. Perturbation Results. We begin by defining some useful quantities. Consider an irreducible stochastic $n \times n$ matrix $T$, and for each pair of indices $1 \leq i, j \leq n$, define $\alpha(i, j)=\min \left\{Q_{l, j}^{\#} \mid T_{i, l}>0\right\}$ and $\beta(i, j)=\max \left\{Q_{l, j}^{\#} \mid T_{i, l}>0\right\}$.

Our first lemma will be helpful in establishing our results.

LEMMA 2.1. Let $T$ be an $n \times n$ irreducible stochastic matrix, and suppose that $E \in \mathcal{E}_{T}$. Then for each positive vector $p^{T}$ such that $p^{T} \mathbf{1}=1$, we have

$$
\left|p^{T} E Q^{\#} e_{j}\right| \leq \frac{\|E\|_{\infty}}{2} \max \left\{Q_{j, j}^{\#}-\sum_{i=1}^{n} p_{i} \alpha(i, j), \sum_{i=1}^{n} p_{i} \beta(i, j)-\min _{1 \leq l \leq n} Q_{l, j}^{\#}\right\}
$$

Proof. Without loss of generality, we take $j=n$. We begin by observing that since $T+E$ is stochastic, $E_{l, m}<0$ only if $T_{l, m}>0$. Fix an index $i$ with $1 \leq i \leq n$, and note that $e_{i}^{T} E$ can be written as $x^{T}-y^{T}$ where each of $x$ and $y$ is nonnegative, where $x^{T} \mathbf{1}=y^{T} \mathbf{1} \leq\|E\|_{\infty} / 2$, and where $y_{l}>0$ only if $T_{i, l}>0$. Thus we have $e_{i}^{T} E Q^{\#} e_{n}=x^{T} Q^{\#} e_{n}-y^{T} Q^{\#} e_{n}=\sum_{l=1}^{n} x_{l} Q_{l, n}^{\#}-\sum_{l=1}^{n} y_{l} Q_{l, n}^{\#}$. It turns out that $Q_{l, n}^{\#}$ is uniquely maximized when $l=n$ (see Theorem 8.5.2 of [2], for example), so it follows that $e_{i}^{T} E Q^{\#} e_{n} \leq \sum_{l=1}^{n} x_{l} Q_{n, n}^{\#}-\sum_{l=1}^{n} y_{l} \alpha(i, n) \leq\left(Q_{n, n}^{\#}-\alpha(i, n)\right)\|E\|_{\infty} / 2$. We also have $e_{i}^{T} E Q^{\#} e_{n}=\sum_{l=1}^{n} x_{l} Q_{l, n}^{\#}-\sum_{l=1}^{n} y_{l} Q_{l, n}^{\#} \geq \sum_{l=1}^{n} x_{l} \min _{1 \leq l \leq n} Q_{l, n}^{\#}-$ $\sum_{l=1}^{n} y_{l} \beta(i, n) \geq\left(\min _{1 \leq l \leq n} Q_{l, n}^{\#}-\beta(i, n)\right)\|E\|_{\infty} / 2$. 
It now follows that $p^{T} E Q^{\#} e_{n} \leq \frac{\|E\|_{\infty}}{2}\left(Q_{n, n}^{\#}-\sum_{i=1}^{n} p_{i} \alpha(i, n)\right)$ and that

$$
p^{T} E Q^{\#} e_{n} \geq-\frac{\|E\|_{\infty}}{2}\left(\beta(i, n)-\min _{1 \leq l \leq n} Q_{l, n}^{\#}\right) .
$$

The result now follows readily.

As mentioned in the introduction, the following inequality appears in [4] and (more explicitly) in [10]. However, neither of those papers characterizes the equality case, as we do below.

THEOREM 2.2. Let $T$ be an $n \times n$ irreducible stochastic matrix, and suppose that $E \in \mathcal{E}_{T}$. Let $\tilde{T}=T+E$, and denote the stationary distributions for $T$ and $\tilde{T}$ by $\pi^{T}$ and $\tilde{\pi}^{T}$, respectively. Then

$$
\left\|\tilde{\pi}^{T}-\pi^{T}\right\|_{\infty} \leq \frac{\|E\|_{\infty}}{2} \max _{1 \leq j \leq n}\left\{Q_{j, j}^{\#}-\min _{1 \leq i \leq n} Q_{i, j}^{\#}\right\}=\kappa(T)\|E\|_{\infty} .
$$

Equality holds if and only if there are indices $j_{0}$ and $a_{1}, \ldots, a_{m}$ such that:

i) for $l=1, \ldots, m, Q_{j_{0}, j_{0}}^{\#}-Q_{a_{l}, j_{0}}^{\#}=\max _{1 \leq j \leq n}\left\{Q_{j, j}^{\#}-\min _{1 \leq i \leq n} Q_{i, j}^{\#}\right\}$;

ii) either $T e_{j_{0}}>0$, or $T\left(e_{a_{1}}+\ldots+e_{a_{m}}\right)>0$;

iii) $E$ can be written as $E=\epsilon\left(\mathbf{1} e_{j_{0}}^{T}-A\right)$, where $A$ is stochastic and has positive entries only in columns $a_{1}, \ldots, a_{m}$, and where $\epsilon$ is chosen so that $T+E$ is nonnegative.

Proof. Since $\tilde{\pi}^{T} Q=\tilde{\pi}^{T} E$, we find upon post-multiplying both sides by $Q^{\#}$ and using the fact that $Q Q^{\#}=I-\mathbf{1} \pi^{T}$, that $\tilde{\pi}^{T}-\pi^{T}=\tilde{\pi}^{T} E Q^{\#}$. Applying Lemma 2.1 we find that for each $j=1, \ldots, n$,

$$
\left|\tilde{\pi}_{j}-\pi_{j}\right| \leq \frac{\|E\|_{\infty}}{2} \max \left\{Q_{j, j}^{\#}-\sum_{i=1}^{n} \tilde{\pi}_{i} \alpha(i, j), \sum_{i=1}^{n} \tilde{\pi}_{i} \beta(i, j)-\min _{1 \leq l \leq n} Q_{l, j}^{\#}\right\}
$$

For each $i, \min _{1 \leq l \leq n} Q_{l, j}^{\#} \leq \alpha(i, j)$ and $\beta(i, j) \leq Q_{j, j}^{\#}$; it now follows that $\| \tilde{\pi}^{T}-$ $\pi^{T}\left\|_{\infty} \leq \frac{\|E\|_{\infty}}{2} \max _{1 \leq j \leq n}\left\{Q_{j, j}^{\#}-\min _{1 \leq i \leq n} Q_{i, j}^{\#}\right\}=\right\| E \|_{\infty} \kappa(T)$.

Suppose now that equality holds, say with $\left|\tilde{\pi}_{j_{0}}-\pi_{j_{0}}\right|=|| \tilde{\pi}^{T}-\pi^{T} \|_{\infty}$. Then in particular we have either $\tilde{\pi}_{j_{0}}-\pi_{j_{0}}=Q_{j_{0}, j_{0}}^{\#}-\sum_{i=1}^{n} \tilde{\pi}_{i} \alpha\left(i, j_{0}\right)=Q_{j_{0}, j_{0}}^{\#}-\min _{1 \leq i \leq n} Q_{i, j_{0}}^{\#}$ or $\tilde{\pi}_{j_{0}}-\pi_{j_{0}}=-\sum_{i=1}^{n} \tilde{\pi}_{i} \beta\left(i, j_{0}\right)+\min _{1 \leq l \leq n} Q_{l, j_{0}}^{\#}=-\left(Q_{j_{0}, j_{0}}^{\#}-\min _{1 \leq i \leq n} Q_{i, j_{0}}^{\#}\right)$. In the former case, referring to the proof of Lemma 2.1, we find that for each $i$, we have $E_{i, j_{0}}=\|E\|_{\infty} / 2$, and further that if $E_{i, a}<0$, then necessarily $Q_{a, j_{0}}^{\#}=$ $\min _{1 \leq l \leq n} Q_{l, j_{0}}^{\#}$. In the latter case, again referring to the proof of Lemma 2.1, we see that for each $i, E_{i, j_{0}}=-\|E\|_{\infty} / 2$, and further that if $E_{i, a}>0$, then necessarily $Q_{a, j_{0}}^{\#}=\min _{1 \leq l \leq n} Q_{l, j_{0}}^{\#}$. Properties i)-iii) are now readily deduced.

Finally we note that the sufficiency of conditions i)-iii) is straightforward to establish. $\square$

The following is immediate.

COROLLARY 2.3. T admits a perturbation $E \in \mathcal{E}_{T}$ such that $\left\|\tilde{\pi}^{T}-\pi^{T}\right\|_{\infty}=$ $\kappa(T)\|E\|_{\infty}$ if and only if there are indices $j_{0}$ and $a_{1}, \ldots, a_{m}$ such that:

i) for each $l=1, \ldots, m, Q_{j_{0}, j_{0}}^{\#}-Q_{a_{l}, j_{0}}^{\#}=2 \kappa(T)$; and

ii) either $T e_{j_{0}}>0$ or $T\left(e_{a_{1}}+\ldots+e_{a_{m}}\right)>0$. 
COROLLARY 2.4. If T has at most one column containing zero entries, then $T$ admits a perturbation $E \in \mathcal{E}_{T}$ so that $\left\|\tilde{\pi}^{T}-\pi^{T}\right\|_{\infty}=\kappa(T)\|E\|_{\infty}$.

Proof. Let $j_{0}$ denote a column index for which $\left|\tilde{\pi}_{j_{0}}-\pi_{j_{0}}\right|=\left\|\tilde{\pi}^{T}-\pi^{T}\right\|_{\infty}$. The conclusion certainly holds if $T e_{j_{0}}$ is positive, so suppose that column has a zero entry. Then all remaining columns of $T$ are positive, and so for each $a_{l}$ such that $Q_{a_{l}, j_{0}}^{\#}$ is minimal, $T e_{a_{l}}>0$. In either case, Corollary 2.3 applies, yielding the result. $\square$

EXAMPLE 2.5. Let $T$ be the $n \times n$ transition matrix given by $T=\frac{1}{n-1}(J-I)$. It follows that $Q^{\#}=\frac{n-1}{n} I-\frac{n-1}{n^{2}} J$ so that in particular, $\kappa(T)=\frac{n-1}{2 n}$. Further, for each index $j$, and for any $i \neq j, Q_{i, j}^{\#}$ is minimal. As a result, even though $T$ has a 0 in each column, there is still a perturbation matrix $E \in \mathcal{E}_{T}$ so that equality holds in (2.1).

Next, we develop the notion of the derivative of the stationary vector with respect to a perturbation matrix. Suppose that $T$ is an irreducible stochastic matrix, and fix a perturbation matrix $E \in \mathcal{E}_{T}$. Observe that for all sufficiently small $\epsilon>0$, the matrix $T_{\epsilon}=T+\epsilon E$ is also irreducible and stochastic. Let $\pi^{T}(\epsilon)$ be the stationary distribution vector for $T_{\epsilon}$, and note that $\pi^{T}(\epsilon)$ is continuous at $\epsilon=0$. We define $\frac{d \pi^{T}}{d E}$ by the following:

$$
\frac{d \pi^{T}}{d E}=\lim _{\epsilon \rightarrow 0^{+}} \frac{\pi^{T}(\epsilon)-\pi^{T}}{\epsilon} .
$$

(Of necessity, our limit is one-sided with respect to $\epsilon$, since if $E \in \mathcal{E}_{T}$ then $T-\epsilon E$ may have negative entries for any positive $\epsilon$.) Since $\epsilon \pi^{T}(\epsilon) E Q^{\#}=\pi^{T}(\epsilon)-\pi^{T}$, it follows that

$$
\frac{d \pi^{T}}{d E}=\pi^{T} E Q^{\#}
$$

Lemma 2.1 is key to the proof of the following result.

THEOREM 2.6. Let $T$ be an $n \times n$ irreducible stochastic matrix. For each $E \in \mathcal{E}_{T}$, we have

$$
\left|\frac{d \pi_{j}}{d E}\right| \leq \frac{\|E\|_{\infty}}{2} \max \left\{Q_{j, j}^{\#}-\sum_{i=1}^{n} \pi_{i} \alpha(i, j), \sum_{i=1}^{n} \pi_{i} \beta(i, j)-\min _{1 \leq l \leq n} Q_{l, j}^{\#}\right\}
$$

Further, there is an $E_{0} \in \mathcal{E}_{T}$ such that equality holds in (2.2). In particular, for all sufficiently small positive $\epsilon$, we have

i) $\epsilon E_{0} \in \mathcal{E}_{T}$, and

ii) letting $\pi^{T}(\epsilon)$ denote the stationary distribution for $T+\epsilon E_{0}$, we have $\left|\pi_{j}(\epsilon)-\pi_{j}\right|=$ $\frac{\left\|\epsilon E_{0}\right\|_{\infty}}{2} \max \left\{Q_{j, j}^{\#}-\sum_{i=1}^{n} \pi_{i} \alpha(i, j), \sum_{i=1}^{n} \pi_{i} \beta(i, j)-\min _{1 \leq l \leq n} Q_{l, j}^{\#}\right\}+o(\epsilon)$.

Proof. Fix $E \in \mathcal{E}_{T}$; we see that for all sufficiently small $\epsilon>0, \pi^{T}(\epsilon)-\pi^{T}=$ $\epsilon \pi^{T}(\epsilon) E Q^{\#}$. Applying Lemma 2.1, we thus find that

$$
\left|\pi_{j}(\epsilon)-\pi_{j}\right| \leq \epsilon \frac{\|E\|_{\infty}}{2} \max \left\{Q_{j, j}^{\#}-\sum_{i=1}^{n} \pi(\epsilon)_{i} \alpha(i, j), \sum_{i=1}^{n} \pi(\epsilon)_{i} \beta(i, j)-\min _{1 \leq l \leq n} Q_{l, j}^{\#}\right\} .
$$


Dividing both sides by $\epsilon$ and letting $\epsilon \rightarrow 0^{+}$yields (2.2).

Let $\tau$ be the smallest positive entry in $T$, and fix an index $j=1, \ldots, n$. Suppose first that $Q_{j, j}^{\#}-\sum_{i=1}^{n} \pi_{i} \alpha(i, j)>\sum_{i=1}^{n} \pi_{i} \beta(i, j)-\min _{1 \leq l \leq n} Q_{l, j}^{\#}$. For each index $i$, select an index $l(i)$ so that $T_{i, l(i)}>0$ and $Q_{l(i), j}^{\#}=\alpha(i, j)$ Let $E_{1}=\sum_{i=1}^{n} e_{i}\left(e_{j}^{T}-e_{l(i)}^{T}\right)$, and observe that if we set $E_{0}=\frac{\tau}{2} E_{1}$, then $E_{0} \in \mathcal{E}_{T}$. It is straightforward to determine that

$$
\frac{d \pi_{j}}{d E_{0}}=\frac{\left\|E_{0}\right\|_{\infty}}{2}\left(Q_{j, j}^{\#}-\sum_{i=1}^{n} \pi_{i} \alpha(i, j)\right)
$$

Now suppose that $Q_{j, j}^{\#}-\sum_{i=1}^{n} \pi_{i} \alpha(i, j) \leq \sum_{i=1}^{n} \pi_{i} \beta(i, j)-\min _{1 \leq l \leq n} Q_{l, j}^{\#}$. For each $i$, find an index $p(i)$ such that $T_{i, p(i)}>0$ and $Q_{p(i), j}^{\#}=\beta(i, j)$. Let $m$ be an index such that $Q_{m, j}^{\#}=\min _{1 \leq l \leq n} Q_{l, j}^{\#}$. Now let $E_{2}=\sum_{i=1}^{n} e_{i}\left(e_{m}^{T}-e_{l(i)}^{T}\right)$. Set $E_{0}=\frac{\tau}{2} E_{2}$; as above, $E_{0} \in \mathcal{E}_{T}$, and

$$
\frac{d \pi_{j}}{d E_{0}}=-\frac{\left\|E_{0}\right\|_{\infty}}{2}\left(\sum_{i=1}^{n} \pi_{i} \beta(i, j)-\min _{1 \leq l \leq n} Q_{l, j}^{\#}\right) .
$$

We conclude that in either case, $E_{0}$ yields equality in (2.2).

Finally note that for all sufficiently small positive $\epsilon$, the matrix $\epsilon E_{0}$ satisfies properties i) and ii). $\square$

EXAMPLE 2.7. Consider the $n \times n$ transition matrix $T$ given by

$$
T=\left[\begin{array}{c|c}
0 & \mathbf{1} \\
\hline \frac{1}{n-1} \mathbf{1}^{T} & 0
\end{array}\right]
$$

and note that $T$ is the transition matrix for a random walk on the star on $n$ vertices. We find that $\pi^{T}=\left[\frac{1}{2 n-2} \mathbf{1}^{T} \mid \frac{1}{2}\right]$, and it is straightforward to verify that

$$
Q^{\#}=\left[\begin{array}{c|c}
I-\frac{3}{4 n-4} J & -\frac{1}{4} \mathbf{1} \\
\hline-\frac{1}{4 n-4} \mathbf{1}^{T} & \frac{1}{4}
\end{array}\right]
$$

A direct computation now shows that $\max \left\{Q_{j, j}^{\#}-\sum_{i=1}^{n} \pi_{i} \alpha(i, j), \sum_{i=1}^{n} \pi_{i} \beta(i, j)-\right.$ $\left.\min _{1 \leq l \leq n} Q_{l, j}^{\#}\right\}=1-\frac{1}{4(n-1)^{2}}$. Thus we see that there are perturbation matrices $E$ of arbitrary small norm such that (letting $\tilde{\pi}^{T}$ be the stationary distribution of $T+E$ )

$$
\left\|\tilde{\pi}^{T}-\pi^{T}\right\|_{\infty} \geq \frac{1}{2}\left(1-\frac{1}{4(n-1)^{2}}\right)\|E\|_{\infty}+o\left(\|E\|_{\infty}\right) .
$$

Theorem 2.2 shows that for any perturbation matrix $E,\left\|\tilde{\pi}^{T}-\pi^{T}\right\|_{\infty} \leq \frac{1}{2}\|E\|_{\infty}$ since $\kappa(T)=\frac{1}{2}$; from Corollary 2.3, that inequality is strict. So while equality cannot hold in (2.1) for the matrix $T$, we find that if $n$ is large, then there is a family of perturbation matrices $E$ so that $\left\|\tilde{\pi}^{T}-\pi^{T}\right\|_{\infty}$ is close to the upper bound in (2.1). 
Motivated by (2.2), we make the following definitions: for each $j=1, \ldots, n$ set

$$
\mu_{j} \equiv Q_{j, j}^{\#}-\sum_{i=1}^{n} \pi_{i} \alpha(i, j)
$$

and

$$
\nu_{j} \equiv \sum_{i=1}^{n} \pi_{i} \beta(i, j)-\min _{1 \leq l \leq n} Q_{l, j}^{\#}
$$

REMARK 2.8. As is noted by Cho and Meyer [3], if $i \neq j$, then $Q_{j, j}^{\#}-Q_{i, j}^{\#}=$ $\pi_{j} m_{i, j}$, where $m_{i, j}$ is the mean first passage time from state $i$ to state $j$. Defining $m_{j, j}$ to be 0 , (this is somewhat unconventional, but useful notation in our context) we thus arrive at another interpretation of the relevant quantities in (2.2): for each $j=1, \ldots, n, \mu_{j}=\pi_{j}\left(\sum_{i=1}^{n} \pi_{i} \max \left\{m_{l, j} \mid T_{i, l}>0\right\}\right)$ and $\nu_{j}=\pi_{j}\left(\max _{1 \leq p \leq n} m_{p, j}-\right.$ $\left.\sum_{i=1}^{n} \pi_{i} \min \left\{m_{l, j} \mid T_{i, l}>0\right\}\right)$. Consequently, we see that $\mu_{j}$ and $\nu_{j}$ depend explicitly on both the mean first passage times for the chain and on the combinatorial properties of the transition matrix.

COROLLARY 2.9. Suppose that $T$ is $n \times n$, irreducible and stochastic. Fix an index $j$. We have

$$
\max _{E \in \mathcal{E}_{T}}\left\{\frac{1}{\|E\|_{\infty}}\left|\frac{d \pi_{j}}{d E}\right|\right\} \geq \frac{1}{4}\left(Q_{j, j}^{\#}-\min _{1 \leq l \leq n} Q_{l, j}^{\#}\right) .
$$

Equality holds in (2.3) if and only if $T$ is a periodic matrix, and one of the equivalence classes of indices in the periodic normal form for $T$ is comprised of the single index $j$.

Proof. From Theorem 2.6 we see that $\max _{E \in \mathcal{E}_{T}} \frac{1}{\|E\|_{\infty}}\left|\frac{d \pi_{j}}{d E}\right|=\frac{1}{2} \max \left\{\mu_{j}, \nu_{j}\right\}$. The inequality (2.3) now follows upon observing that $\max \left\{\mu_{j}, \nu_{j}\right\} \geq \frac{1}{2}\left(\mu_{j}+\nu_{j}\right)=$ $\frac{1}{2}\left(Q_{j, j}^{\#}-\sum_{i=1}^{n} \pi_{i} \alpha(i, j)+\sum_{i=1}^{n} \pi_{i} \beta(i, j)-\min _{1 \leq l \leq n} Q_{l, j}^{\#}\right)=\frac{1}{2}\left(Q_{j, j}^{\#}-\min _{1 \leq l \leq n} Q_{l, j}^{\#}+\right.$ $\left.\sum_{i=1}^{n} \pi_{i}(\beta(i, j)-\alpha(i, j))\right) \geq \frac{1}{2}\left(Q_{j, j}^{\#}-\min _{1 \leq l \leq n} Q_{l, j}^{\#}\right)$. Next suppose that equality holds in (2.3) and without loss of generality we take the index $j$ to be $n$. Fix an index $i$ between 1 and $n$. Necessarily we have $\alpha(i, n)=\beta(i, n)$, so that either there is a single index $l$ such that $T_{i, l}>0$ (in which case $T_{i, l}=1$ ) or for any pair of indices $l_{1}, l_{2}$ such that $T_{i, l_{1}}, T_{i, l_{2}}>0$, we have $Q_{l_{1}, n}^{\#}=Q_{l_{2}, n}^{\#}$. From this observation it follows that if $T_{i, l}>0$, then $e_{i}^{T}\left(I-\mathbf{1} \pi^{T}\right) e_{n}=e_{i}^{T}(I-T) Q^{\#} e_{n}=e_{i}^{T} Q^{\#} e_{n}-e_{i}^{T} T Q^{\#} e_{n}=Q_{i, n}^{\#}-Q_{l, n}^{\#}$. In particular, if $i \neq n$, we deduce that $Q_{i, n}^{\#}=Q_{l, n}^{\#}-\pi_{n}$.

Consider the directed graph on vertices $1, \ldots, n$, corresponding to $T$. We claim that if $i$ is a vertex at distance $d \geq 1$ from $n$, then $Q_{i, n}^{\#}=Q_{n, n}^{\#}-d \pi_{n}$. In order to establish the claim, we use induction on $d$. If $d=1$, then $T_{i, n}>0$ and hence $Q_{i, n}^{\#}=Q_{n, n}^{\#}-\pi_{n}$, as desired. Suppose now that the claim holds for vertices at distance $d$ from $n$, and that the distance from $i$ to $n$ is $d+1$. Then in particular, there is a vertex $l$ at distance $d$ from $n$ such that $T_{i, l}>0$. Hence we have $Q_{i, n}^{\#}=$ $Q_{l, n}^{\#}-\pi_{n}=Q_{n, n}^{\#}-d \pi_{n}-\pi_{n}=Q_{n, n}^{\#}-(d+1) \pi_{n}$, completing the induction step. From the claim we now deduce that if $i$ is at distance $d$ from $n$, then $T_{i, l}>0$ only 
if $l$ is at distance $d-1$ from $n$. It now follows that we can partition the vertices in $\{1, \ldots, n-1\}$ into subsets $S_{1}, \ldots, S_{k}$ such that $i \in S_{l}$ if and only if the distance from $i$ to $n$ is $l$, and such that if $a \neq n, T_{a, b}>0$ only if for some $l$ between 2 and $k, a \in S_{l}$ and $b \in S_{l-1}$. Evidently $a \in S_{l}$ if and only if $Q_{a, n}^{\#}=Q_{n, n}^{\#}-l \pi_{n}$; from this last we see that there is an index $l$ such that $T_{n, a}>0$ only if $a \in S_{l}$, and since $T$ is irreducible, necessarily $l=k$. Consequently, $T$ is a periodic matrix, and one of the equivalence classes of indices in the periodic normal form for $T$ is comprised of the single index $n$.

Finally, if $T$ is periodic, with period $p$ say and has $\{n\}$ as a single equivalence class, then a result of Kirkland [7] shows that $Q_{n, n}^{\#}=\frac{p-1}{2 p}$ while if $i$ is at distance $d$ from $n$, then $Q_{i, n}^{\#}=\frac{p-1}{2 p}-\frac{d}{p}$. It now follows that equality holds in (2.3).

Next, we make another connection between $\left\|\tilde{\pi}^{T}-\pi^{T}\right\|_{\infty}$ and $\kappa(T)$.

THEOREM 2.10. Let $T$ be an $n \times n$ irreducible stochastic matrix with stationary vector $\pi^{T}$. Fix an index $j=1, \ldots, n$. There is a matrix $E$ such that for all sufficiently small positive $\epsilon$, we have $\epsilon E \in \mathcal{E}_{T}$, and further, the stationary vector, $\pi^{T}(\epsilon)$, of $T+\epsilon E$ satisfies $\left|\pi_{j}(\epsilon)-\pi_{j}\right|>\frac{1}{4}|| \epsilon E \|_{\infty}\left(Q_{j, j}^{\#}-\min _{1 \leq l \leq n} Q_{l, j}^{\#}\right)$.

Proof. Applying Corollary 2.9, we see that the result follows immediately if (2.3) is strict. If equality holds in (2.3) then, taking $j=n$, we find that $T$ can be written as

$$
T=\left[\begin{array}{cccccc}
0 & T_{1} & 0 & 0 & \ldots & 0 \\
0 & 0 & T_{2} & 0 & \ldots & 0 \\
\vdots & & & \ddots & & \vdots \\
0 & 0 & & \ldots & 0 & \mathbf{1} \\
t_{p}^{T} & 0 & 0 & \ldots & 0 & 0
\end{array}\right]
$$

It is straightforward to check that

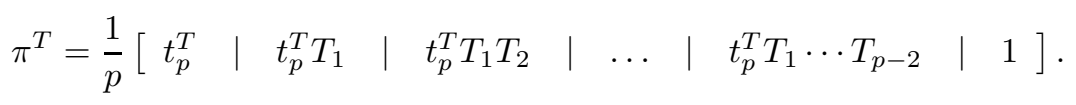

In particular, we have $\pi_{n}=\frac{1}{p}$ and that $Q_{n, n}^{\#}-\min _{1 \leq l \leq n} Q_{l, n}^{\#}=\frac{p-1}{2 p}$. Next consider the following perturbation matrix:

$$
E=\left[\begin{array}{cccccc}
0 & -A_{1} & 0 & 0 & \ldots & \mathbf{1} \\
0 & 0 & -A_{2} & 0 & \ldots & \mathbf{1} \\
\vdots & & & \ddots & & \vdots \\
0 & 0 & & \ldots & 0 & 0 \\
-a_{p}^{T} & 0 & 0 & \ldots & 0 & 1
\end{array}\right]
$$

where each $A_{i}$ satisfies $A_{i} \mathbf{1}=\mathbf{1}$ and has positive entries only in positions for which $T_{i}$ is positive, and where $a_{p}^{T} \mathbf{1}=1$ with $a_{p}^{T}$ positive only where $t_{p}^{T}$ is positive. We see that for all sufficiently small positive $\epsilon, T+\epsilon E$ is irreducible and stochastic, and it can be shown that

$$
\pi^{T}(\epsilon)=\frac{\epsilon}{1-(1-\epsilon)^{p}}\left[u^{T} \quad\left|\quad u^{T} S_{1} \quad\right| \quad u^{T} S_{1} S_{2} \quad|\quad \ldots \quad| u^{T} S_{1} \cdots S_{p-2} \mid 1\right]
$$


where $u^{T}=t_{p}^{T}+\epsilon a_{p}^{T}$ and $S_{i}=T_{i}+\epsilon A_{i}$ for each $i=1, \ldots, p-2$.

Hence

$$
\pi_{n}(\epsilon)-\pi_{n}=\frac{p \epsilon+(1-\epsilon)^{p}-1}{p\left(1-(1-\epsilon)^{p}\right)} .
$$

From the fact that

$$
(1-\epsilon)^{p}=1-p \epsilon+\frac{p(p-1)}{2} \epsilon^{2}-\frac{p(p-1)(p-2)}{6} \epsilon^{3}+o\left(\epsilon^{3}\right),
$$

we find that for all sufficiently small $\epsilon>0$,

$$
\frac{p \epsilon+(1-\epsilon)^{p}-1}{p\left(1-(1-\epsilon)^{p}\right)}>\frac{p-1}{2 p} \epsilon=\frac{1}{4}\|\epsilon E\|_{\infty}\left(Q_{n, n}^{\#}-\min _{1 \leq l \leq n} Q_{l, n}^{\#}\right) . \square
$$

The following is a direct consequence of Theorem 2.10.

COROLLARY 2.11. Let $T$ be an irreducible stochastic matrix with stationary vector $\pi^{T}$. There is a matrix $E \in \mathcal{E}_{T}$ such that for all sufficiently small positive $\epsilon$, the stationary vector, $\pi^{T}(\epsilon)$, of $T+\epsilon E$ satisfies $\left\|\pi^{T}(\epsilon)-\pi^{T}\right\|_{\infty}>\|\epsilon E\|_{\infty} \frac{\kappa(T)}{2}$.

REMARK 2.12. Taken together, Theorem 2.2 and Corollary 2.11 show that the quantity $\kappa(T)$ is of the right order of magnitude in terms of measuring the sensitivity of the stationary vector. The former shows $\left\|\tilde{\pi}^{T}-\pi^{T}\right\|_{\infty} \leq \kappa(T)\|E\|_{\infty}$ for any $E \in \mathcal{E}_{T}$, while the latter shows that there is a family of perturbation matrices $E \in \mathcal{E}_{T}$ such that for each member of that family, $\left\|\tilde{\pi}^{T}-\pi^{T}\right\|_{\infty}>\kappa(T)\|E\|_{\infty} / 2$.

COROLLARY 2.13. Let $T$ be an $n \times n$ irreducible stochastic matrix. Fix an index $j$; for each $\epsilon>0$, there is a perturbation matrix $E \in \mathcal{E}_{T}$ with $\|E\|_{\infty}<\epsilon$ such that the stationary vector $\tilde{\pi}^{T}$ for the irreducible stochastic matrix $T+E$ satisfies $\left|\tilde{\pi}_{j}-\pi_{j}\right|>\|E\|_{\infty} / 8$.

Proof. In [8] it is shown that for each $j, Q_{j, j}^{\#}-\min _{1 \leq l \leq n} Q_{l, j}^{\#} \geq \frac{1}{2}$. The conclusion now follows from Theorem 2.10.

Corollary 2.13 yields the following.

COROLLARY 2.14. Let $T$ be an $n \times n$ irreducible stochastic matrix. For each $\epsilon>0$, there is a perturbation matrix $E \in \mathcal{E}_{T}$ with $\|E\|_{\infty}<\epsilon$ such that the stationary vector $\tilde{\pi}^{T}$ for the irreducible stochastic matrix $T+E$ satisfies $\max _{1 \leq j \leq n} \frac{\left|\tilde{\pi}_{j}-\pi_{j}\right|}{\pi_{j}}>$ $\|E\|_{\infty} \frac{n}{8}$.

Proof. Since the entries of $\pi^{T}$ sum to 1 , we see that there is at least one index $j$ such that $\pi_{j} \leq \frac{1}{n}$. For that index $j$, there is an $E \in \mathcal{E}_{T}$ such that $\left|\tilde{\pi}_{j}-\pi_{j}\right|>\|E\|_{\infty} / 8$, by Corollary 2.13. Consequently, $\frac{\left|\tilde{\pi}_{j}-\pi_{j}\right|}{\pi_{j}}>\|E\|_{\infty} \frac{n}{8}$, yielding the result.

REMARK 2.15. In [5], Ipsen and Meyer investigate the notion of relative stability for the stationary vector. Corollary 2.14 shows that for large values of $n$, there is always at least one index $j$ and a family of admissible perturbation matrices such that for each member $E$ of that family, the relative error $\frac{\left|\tilde{\pi}_{j}-\pi_{j}\right|}{\pi_{j}}$ is large compared to $\|E\|_{\infty}$

Our final result of this section provides, for certain perturbation matrices $E$, a lower bound on $\left\|\tilde{\pi}^{T}-\pi^{T}\right\|_{\infty}$ in terms of the minimum diagonal entry of $T$. 
COROLLARY 2.16. Let $T$ be an $n \times n$ irreducible stochastic matrix with minimum diagonal entry a. There is a perturbation matrix $E \in \mathcal{E}_{T}$ such that for each $0<\epsilon \leq 1$, the stationary vector $\tilde{\pi}^{T}$ for the irreducible stochastic matrix $T+\epsilon E$ satisfies $\left\|\tilde{\pi}^{T}-\pi^{T}\right\|_{\infty}>\|\epsilon E\|_{\infty} \frac{n-1}{4 n(1-a)}$.

Proof. Since the minimum diagonal entry of $T$ is $a$, we can write $T$ as $T=$ $a I+(1-a) S$, where $S$ is irreducible and stochastic. Then $Q=I-T=(1-a)(I-$ $S)$, and it follows that $Q^{\#}=\frac{1}{1-a} A^{\#}$, where $A=I-S$. In [8] it is shown that $\kappa(S) \geq \frac{n-1}{2 n}$ and hence we find that $\kappa(T) \geq \frac{n-1}{2 n(1-a)}$. Select an index $j$ for which $\frac{1}{2}\left(Q_{j, j}^{\#}-\min _{1 \leq i \leq n} Q_{i, j}^{\#}\right)=\kappa(T)$, and then select an $E \in \mathcal{E}_{T}$ so that for the family of matrices $T+\epsilon E,\left|\pi_{j}(\epsilon)-\pi_{j}\right|>\frac{\kappa(T)\|\epsilon E\|_{\infty}}{2}$. The result now follows.

3. Conditioning for a Random Walk on a Tree. It is natural wonder whether a transition matrix with stationary vector $\pi^{T}$ has the property that for each perturbation matrix $E,\left\|\tilde{\pi}^{T}-\pi^{T}\right\|_{\infty} \leq\|E\|_{\infty}$, or whether $T$ admits a perturbation $E \in \mathcal{E}_{T}$ so that $\left\|\tilde{\pi}^{T}-\pi^{T}\right\|_{\infty}>\|E\|_{\infty}$. In the former case we can consider $\pi^{T}$ to be stable under perturbation, while in the latter case we may think of $\pi^{T}$ as being unstable, in the sense that it is possible for the norm of $\tilde{\pi}^{T}-\pi^{T}$ to magnify the norm of the perturbing matrix.

To be more precise, we say that the stationary vector $\pi^{T}$ for an irreducible transition matrix $T$ is globally well-conditioned if, for any perturbation matrix $E \in$ $\mathcal{E}_{T},\left\|\tilde{\pi}^{T}-\pi^{T}\right\|_{\infty} \leq\|E\|_{\infty}$, and we say that $\pi^{T}$ is locally well-conditioned if there is an $\epsilon>0$ such that for each perturbation matrix $E \in \mathcal{E}_{T}$ such that $\|E\|_{\infty}<$ $\epsilon,\left\|\tilde{\pi}^{T}-\pi^{T}\right\|_{\infty} \leq\|E\|_{\infty}$. Finally, we say that $\pi^{T}$ is poorly conditioned if for each $\epsilon>0$, there is a perturbation matrix $E \in \mathcal{E}_{T}$ such that $\|E\|_{\infty}<\epsilon$, and $\left\|\tilde{\pi}^{T}-\pi^{T}\right\|_{\infty}>\|E\|_{\infty}$. Note that by Theorem 2.2, $\pi^{T}$ is globally well-conditioned if $\kappa(T) \leq 1$, while by Corollary 2.11 , if $\kappa(T) \geq 2$, then $\pi^{T}$ is poorly conditioned. Also, by Theorem 2.6, we find that $\pi^{T}$ is locally well-conditioned provided that $\mu_{j}, \nu_{j}<2$ for each $j=1, \ldots, n$, while $\pi^{T}$ is poorly conditioned if for some $j$, either $\mu_{j}>2$ or $\nu_{j}>2$.

In this section, we explore these ideas for a restricted class of Markov chains, namely the random walks generated by trees. Specifically, we characterize the trees for which the transition matrix of the corresponding random walk is locally wellconditioned. As is the case for other quantities associated with a random walk on a tree (see [9], for example) combinatorial formulae for the entries in $Q^{\#}$ are available for such transition matrices, thus making the problem tractable.

Suppose that $\mathcal{T}$ is a tree on $n$ vertices with adjacency matrix $A$, and for each $i=1, \ldots, n$, let $d_{i}$ be the degree of vertex $i$; for distinct vertices $i, j$, we let $\delta(i, j)$ denote the distance from $i$ to $j$. Setting $D=\operatorname{diag}\left(d_{1}, \ldots, d_{n}\right)$, the transition matrix for the random walk on $\mathcal{T}$ is given by $T=D^{-1} A$. By an abuse of terminology, we say that $\mathcal{T}$ is globally well-conditioned, locally well-conditioned, or poorly conditioned according as the stationary vector for $T$ is. (Thus by referring to Example 2.7, we see that if $\mathcal{T}$ is a star, then $\mathcal{T}$ is globally well-conditioned.) It is readily verified that the corresponding stationary vector for $T$ is $\pi^{T}=\frac{1}{2(n-1)}\left[\begin{array}{llll}d_{1} & d_{2} & \ldots & d_{n}\end{array}\right]$. Let $m_{i, j}$ be the mean first passage time between vertices $i$ and $j$; we define $m_{i, i}$ to be 0 for each 
$i$. Then for any pair of vertices $i, j$, we find from Remark 2.8 that

$$
Q_{j, j}^{\#}-Q_{i, j}^{\#}=\frac{m_{i, j} d_{j}}{2(n-1)}
$$

Fortunately, there is a combinatorial method for computing the necessary quantities $m_{i, j}$. The following result from [9] gives that method.

PROPOSITION 3.1. Let $\mathcal{T}$ be a tree on $n$ vertices. Suppose that $i$ and $j$ are distinct vertices at distance $\delta(i, j)$, and denote the edges on the path between them by $f_{1}, \ldots, f_{\delta(i, j)}$. For each $l=1, \ldots, \delta(i, j)$, let $\lambda_{l}$ be the number of vertices in the component of $\mathcal{T} \backslash f_{l}$ that contains $i$. Then $m_{i, j}=\sum_{l=1}^{\delta(i, j)}\left(2 \lambda_{l}-1\right)$.

The following is a useful consequence.

COROLLARY 3.2. Let $\mathcal{T}$ be a tree on $n$ vertices, and suppose that $i$ and $j$ are distinct vertices. Then $m_{i, j}+m_{j, i}=2(n-1) \delta(i, j)$.

Proof. Suppose that the edges on the path from $i$ to $j$ are $f_{1}, \ldots, f_{\delta(i, j)}$. For each $l=1, \ldots, \delta(i, j)$, let $\lambda_{l}$ be the number of vertices in the component of $\mathcal{T} \backslash f_{l}$ containing $i$, and let $\lambda_{l}^{\prime}$ denote the number of vertices in the component of $\mathcal{T} \backslash f_{l}$ containing $j$. From Proposition 3.1, we find that $m_{i, j}+m_{j, i}=\sum_{l=1}^{\delta(i, j)}\left(2 \lambda_{l}-1\right)+\sum_{l=1}^{\delta(i, j)}\left(2 \lambda_{l}^{\prime}-1\right)=$ $2 \sum_{l=1}^{\delta(i, j)}\left(\lambda_{l}+\lambda_{l}^{\prime}\right)-2 \delta(i, j)$. The result follows upon observing that $\lambda_{l}+\lambda_{l}^{\prime}=n$ for each $l . \square$

In this section we deal with several subclasses of trees, and we introduce those now.

Suppose that $k \geq 2$ and that we are given $l_{1}, \ldots, l_{k} \in \mathbb{N}$; let $S\left(l_{1}, \ldots, l_{k}\right)$ be the tree on $\sum_{i=1}^{k} l_{i}+1$ vertices formed by taking a central vertex $c_{0}$ and for each $i=1, \ldots, k$, attaching a path on $l_{i}$ vertices at $c_{0}$. Observe that in the case that each $l_{i}$ is 1 , the correspondingly constructed tree is just a star.

Suppose that $k, p \geq 2$, and that we are given parameters $l_{1}, \ldots, l_{k} \in \mathbb{N}$ and $m_{1}, \ldots, m_{p} \in \mathbb{N}$. Let $R\left(\bar{l}_{1}, \ldots, l_{k} ; m_{1}, \ldots, m_{p}\right)$ be the tree on $\sum_{i=1}^{k} l_{i}+\sum_{j=1}^{p} m_{j}+2$ vertices formed by starting with a central edge between vertices $u_{0}$ and $v_{0}$; at vertex $u_{0}$, for each $i=1, \ldots, k$, attach a path on $l_{i}$ vertices, and at vertex $v_{0}$, for each $j=1, \ldots, p$, attach a path on $m_{j}$ vertices. In the resulting tree, $d_{u_{0}}=k+1, d_{v_{0}}=p+1$, and in the special case that each $l_{i}$ and $m_{j}$ is 1 , the tree has diameter 3 .

Suppose that $n_{1}, \ldots, n_{k} \geq 2$ and that we are given parameters $l_{1,1}, \ldots, l_{n_{1}, 1}$, $\ldots, l_{1, k}, \ldots, l_{n_{k}, k} \in \mathbb{N}$ and a nonnegative integer $p$. Let

$T_{p}\left(l_{1,1}, \ldots, l_{n_{1}, 1} ; \ldots ; l_{1, k}, \ldots, l_{n_{k}, k}\right)$ denote the tree on $\left(\sum_{i=1}^{k} \sum_{j=1}^{n_{i}} l_{j, i}\right)+k+p+1$ vertices constructed as follows: start with a star on $k+p+1$ vertices, with central vertex $c_{1}$ and pendant vertices $1, \ldots, k+p$. For each $i=1, \ldots, k$, at the pendant vertex $i$, attach paths of lengths $l_{1, i}, \ldots, l_{n_{i}, i}$. Observe that the resulting tree has the property that if $i$ and $j$ are vertices of degree at least 3 , then $\delta(i, j) \leq 2$.

EXAMPLE 3.3. Consider the tree $\mathcal{T}=S(2, \ldots, 2,1, \ldots, 1)$, where at the central vertex $c_{0}$, there are $r \geq 1$ branches of length 2 and $s$ branches of length 1 ; here we assume that $r+s \geq 2$ and we admit the possibility that $s=0$. It is straightforward to verify that if $u$ is not a next to pendant vertex on a branch of length 2 at $c_{0}$, then for each vertex $w \neq u, \pi_{u} m_{w, u} \leq 2$ with equality if and only if $u$ and $w$ are pendant 
vertices on different branches of length 2 at $c_{0}$. It then follows readily that $\mu_{u}, \nu_{u}<2$. A similar analysis reveals that if $r=1$, then $\mathcal{T}$ is globally well-conditioned.

Suppose now that $r \geq 2$, and note that if $u$ is a next to pendant vertex on a branch of length 2 at $c_{0}$, we have, upon denoting the number of vertices by $n=2 r+s+1$, that $\mu_{u}=\frac{1}{2(n-1)^{2}}\left(4(n-1)^{2}-2(n-r)-3(s+2)\right)<2$, and $\nu_{u}=\frac{1}{8 r^{2}+8 r s+2 s^{2}}\left(4 r^{2}+6 r s+\right.$ $\left.2 s^{2}+22 r+11 s-8\right)<2$. Consequently, we see that $\mathcal{T}$ is locally well-conditioned.

The following result gives some necessary conditions for a tree to be locally wellconditioned.

LEMMA 3.4. Let $\mathcal{T}$ be a locally well-conditioned tree on $n$ vertices. For any pair of nonpendant vertices $i$ and $j$, we have $\delta(i, j) \leq 3$. Further, if $d_{i}, d_{j} \geq 3$, then $\delta(i, j) \leq 2$.

Proof. Suppose that we have distinct vertices $i$ and $j$. Since $\mathcal{T}$ is locally wellconditioned, $d_{j} m_{i, j}<8(n-1)$ and $d_{i} m_{j, i}<8(n-1)$. From Corollary 3.2, we have $m_{i, j}+m_{j, i}=2 \delta(i, j)(n-1)$, so that $\max \left\{m_{i, j}, m_{j, i}\right\} \geq \delta(i, j)(n-1)$. Thus we have $2 \delta(i, j)(n-1) \leq \max \left\{d_{j} m_{i, j}, d_{i} m_{j, i}\right\}<8(n-1)$, from which we conclude that $\delta(i, j) \leq 3$. Further, if $d_{i}, d_{j} \geq 3$, then $3 \delta(i, j)(n-1) \leq \max \left\{d_{j} m_{i, j}, d_{i} m_{j, i}\right\}<8(n-1)$, yielding $\delta(i, j) \leq 2$.

EXAMPLE 3.5. Consider the path on $n$ vertices, $\mathcal{P}_{n}$. From Lemma 3.4 we see that if $n \geq 7$, then $\mathcal{P}_{n}$ is poorly conditioned. We note that $\mathcal{P}_{6}$ is also poorly conditioned, since for that graph, $\mu_{u} / 2=115 / 100>1$, where $u$ is a next to pendant vertex.

Note that by Example 2.7, $\mathcal{P}_{3}$ is globally well-conditioned, and a direct computation reveals that the same is true of $\mathcal{P}_{4}$. Finally, we note that by Example $3.3, \mathcal{P}_{5}$ is locally well-conditioned. Thus we see that $\mathcal{P}_{n}$ is locally well-conditioned if and only if $n \leq 5$.

From Lemma 3.4 we see that a tree $\mathcal{T}$ is a candidate to be locally well-conditioned, then it falls into one of several categories:

i) $\mathcal{T}$ has maximum degree 2. In that case $\mathcal{T}$ is a path, and appealing to Example 3.5, we find that in fact $\mathcal{T}$ is a path on at most 5 vertices.

ii) $\mathcal{T}$ has exactly one vertex of degree at least 3 . In that case, $\mathcal{T}$ is of the form $S\left(l_{1}, \ldots, l_{k}\right)$.

iii) $\mathcal{T}$ has more than one vertex of degree at least 3 , and the distance between any two such vertices is 1 . In that case, there are just two vertices of degree at least 3 , necessarily adjacent, and so $\mathcal{T}$ has the form $R\left(l_{1}, \ldots, l_{k} ; m_{1}, \ldots, m_{p}\right)$.

iv) $\mathcal{T}$ has a pair of vertices $i, j$ such that $d_{i}, d_{j} \geq 3$ and $\delta(i, j)=2$. In that case, $\mathcal{T}$ has the form $T_{p}\left(l_{1,1}, \ldots, l_{n_{1}, 1} ; \ldots ; l_{1, k}, \ldots, l_{n_{k}, k}\right)$.

The following sequence of lemmas deals with the trees in each of the subclasses arising in ii) - iv). We begin with the class $S\left(l_{1}, \ldots, l_{k}\right)$.

LEMMA 3.6. Suppose that $\mathcal{T}$ is a locally well-conditioned tree of the form $S\left(l_{1}, \ldots, l_{k}\right)$, where $k \geq 3$ and $l_{1} \geq \ldots \geq l_{k} \geq 1$. Then $l_{1} \leq 2$.

Proof. From Lemma 3.4, we can see that for each pair of distinct indices $i, j, l_{i}+$ $l_{j}-2 \leq 3$. Consequently, we find that $1 \leq l_{1} \leq 4$. Let $c_{0}$ denote the central vertex (of degree $k$ ).

If $l_{1}=4$, then necessarily each of $l_{2}, \ldots, l_{k}$ must be 1 , and so the number of 
vertices is $k+4$. A straightforward computation shows that $\nu_{c_{0}}=\frac{31 k^{2}+43 k}{4(k+3)^{2}}$, which exceeds 2 since $k \geq 3$. Thus if $\mathcal{T}$ is locally well-conditioned, then in fact $l_{1} \leq 3$.

If $l_{1}=3$ then necessarily $l_{2} \leq 2$. Suppose that in addition to the branch at $c_{0}$ that is a path of length 3 , there are $r$ branches at $c_{0}$ that are paths of length 2 , and $s$ pendant vertices at $c_{0}$; here we admit the possibility that one of $r$ and $s$ is 0 . In particular, the number of vertices is $2 r+s+4$. Suppose first that $r=0$, so that necessarily $s \geq 2$. A straightforward computation shows that $\nu_{c_{0}}=\frac{(s+1)(17 s+35)}{4(s+3)^{2}}$, which is easily seen to exceed 2. Next suppose that $r \geq 1$, and let $u$ be the next to pendant vertex on the branch at $c_{0}$ containing 3 vertices. We find that $\mu_{u}=$ $\frac{32 r^{2}+32 r s+8 s^{2}+62 r+27 s+21}{2(2 r+s+3)^{2}}$, which turns out to exceed 2. In either case we see that if $l_{1}=3$, then $\mathcal{T}$ is poorly conditioned.

We thus conclude that if $\mathcal{T}$ is locally well-conditioned, then $l_{1} \leq 2$.

REMARK 3.7. Together Lemma 3.6, Example 2.7 and Example 3.3 show that a tree of the form $S\left(l_{1}, \ldots, l_{k}\right)$ is locally well-conditioned if and only if $l_{i} \leq 2, i=$ $1, \ldots, k$.

Next, we handle the class $R\left(l_{1}, \ldots, l_{k} ; m_{1}, \ldots, m_{p}\right)$.

LEMMA 3.8. Suppose that $\mathcal{T}=R\left(l_{1}, \ldots, l_{k} ; m_{1}, \ldots, m_{p}\right)$. Then $\mathcal{T}$ is locally well-conditioned if and only if each $l_{i}$ and $m_{j}$ is 1 , and, up to relabeling of $k$ and $p$, either $p=3$ and $3 \leq k \leq 4$ or $p=2$ and $2 \leq k \leq 6$.

Proof. Let the endpoints of $\mathcal{T}$ 's central edge be $u_{0}$ and $v_{0}$, with the paths of length $l_{i}, i=1, \ldots, k$ being attached at $u_{0}$ and the paths of length $m_{j}, j=1, \ldots, p$ being attached at $v_{0}$. Reindexing if necessary, we assume that $l_{1} \geq \ldots \geq l_{k}$ and that $m_{1} \geq \ldots \geq m_{p}$. Applying Lemma 3.4 we find that $m_{1}+l_{1} \leq 4, l_{1}+l_{2} \leq 5$ and $m_{1}+m_{2} \leq 5$. Without loss of generality we take $m_{1} \geq l_{1}$, and we are led to two cases: either $m_{1}=3, m_{2} \leq 2$ and $l_{1}=1$ or $m_{1}, l_{1} \leq 2$.

Suppose first that $m_{1}=3$. Then $\mathcal{T}$ has the form

$R(1, \ldots, 1 ; 3,2, \ldots, 2,1, \ldots, 1)$ where the number of 1 's in the first list of parameters is $k$, the number of 2's in the second list of parameters is $t$, and number of 1 's in the second list of parameters is $q$. In particular the number of vertices is $n=k+q+2 t+5$ and $p=t+q+1$. Let $y$ be the next to pendant vertex on the path of length 3 at vertex $v_{0}$. A computation shows that

$$
\begin{aligned}
\mu_{y}= & \frac{2}{4(n-1)^{2}}\{(t+q+k+2)(4 n+2 k-11)+(k+1)(4 n+2 k-10)+q(4 n-12) \\
& \quad+t(4 n-9)+2 t(4 n-8)+2(4 n-12)+2(2 n-5)\} \\
= & \frac{(n-3)(4 n+2 k-11)+(k+1)(4 n+2 k-10)+12 n-34+t(8 n-2 k-14)+q(4 n-12)}{2(n-1)^{2}} \\
\geq & \frac{4 n^{2}+5 n-31}{2(n-1)^{2}},
\end{aligned}
$$

the inequality following from the fact that $k \geq 2$. Since $\frac{4 n^{2}+5 n-31}{2(n-1)^{2}}>2$, we find that $\mathcal{T}$ is poorly conditioned.

Next, suppose that $m_{1}=2$; we see that $\mathcal{T}$ has the form

$$
R(2, \ldots, 2,1, \ldots, 1 ; 2, \ldots, 2,1, \ldots, 1)
$$


where the number of 2's in the first list of parameters is $t$, the number of 1's in the first list of parameters is $q$, the number of 2's in the second list of parameters is $s$, and number of 1's in the second list of parameters is $r$. In particular, the number of vertices is $n=2 s+2 t+q+r+2, k=t+q$ and $p=r+s$. Let $y$ be a next to pendant vertex on a path of length 2 at $v_{0}$. We find that $\mu_{y}$ equals

$$
\frac{(2 n-4)(2 n-3)+(2 t+q+1)(4 t+2 q+3)+2 t(4 t+2 q+4)+(4 t+2 q)(q+r+s+1)+8 s-r-10}{2(n-1)^{2}} .
$$

It follows that $\mu_{y}>2$ if and only if $(4 t+2 q)(r+q+s+1)+(2 t+q+1)(4 t+2 q+$ $3)+2 t(4 t+2 q+4)>7 r+4 s+12 t+6 q+14$. If $2 t+q \geq 4$, this last inequality is readily seen to hold, so we need only deal with the case that $2 t+q \leq 3$. Recalling that $t+q \geq 2$, we have just three possibilities: $t=q=1 ; t=0, q=2$; and $t=0, q=3$.

If $t=q=1$, then applying an analogous argument at a next to pendant vertex on a branch of length 2 at $u_{0}$, we find that if $2 s+r \geq 4$, then $\mathcal{T}$ is poorly conditioned. The remaining case is that $s=r=1$, and a direct computation reveals that $\mu_{v_{0}}>2$ in that case.

If $t=0$ and $q=2$ then $r \leq 2$ implies that $\mu_{y}>2$, while if $t=0$ and $q=3$, then $r \leq 29$ implies that $\mu_{y}>2$. Suppose now that either $t=0, q=2$ and $r \geq 3$, or $t=0, q=3$ and $r \geq 30$. A computation shows that $\mu_{u_{0}}=\frac{(q+1)}{4(n-1)^{2}}((2 n-2 q-3)(2 n-$ $q-2)+14 s+3 r+3)$. Substituting in $q=2$ or 3 into that last quantity and applying the corresponding lower bounds on $r$, it follows readily that $\mu_{u_{0}}>2$. Consequently, we find that $\mathcal{T}$ is poorly conditioned.

Finally if $m_{1}=1$, then $\mathcal{T}$ is a tree (with diameter 3 ) of the form $R(1, \ldots, 1 ; 1, \ldots, 1$ ), where there are $k 1$ 's in the first list of parameters and $p 1$ 's in the second list of parameters. In particular the number of vertices is $n=k+p+2$, and without loss of generality we assume that $k \geq p \geq 2$. First note that if $x$ and $y$ are pendant vertices, then $\pi_{x} m_{y, x} \leq \frac{2 n+2 k-1}{2 n-2}<2$. Thus in order that $\mathcal{T}$ is locally well-conditioned, we need to determine the values of $\mu_{u_{0}}, \nu_{u_{0}}, \mu_{v_{0}}$ and $\nu_{v_{0}}$. We have $\mu_{u_{0}}=\frac{k+1}{4(n-1)^{2}}((k+$ $1)(2 p+1)+(p+1)(2 p+2)+p(2 p+1))$ and $\nu_{u_{0}}=\frac{k+1}{4(n-1)^{2}}(k(2 p+2)+(k+1)(2 p+1)+$ $(p+1)(2 p+2)+p)$, with analogous expressions holding for $\mu_{v_{0}}$ and $\nu_{v_{0}}$. Since $k \geq p$, it follows that $\mu_{u_{0}} \leq \nu_{u_{0}}$ and that $\mu_{v_{0}} \leq \nu_{v_{0}}$ if $k=p$, while $\mu_{v_{0}}>\nu_{v_{0}}$ if $k \geq p+1$.

In the case that $k=p$ then $\nu_{u_{0}}=\nu_{v_{0}}$, so $\mathcal{T}$ is locally well-conditioned if $\nu_{u_{0}}<$ 2 , and poorly conditioned if $\nu_{u_{0}}>2$. It is readily seen that the former holds if $6 k^{3}-16 k^{2}-19 k-5<0$, and the latter holds if $6 k^{3}-16 k^{2}-19 k-5>0$. We conclude that $\mathcal{T}$ is locally well-conditioned if $k \leq 3$ and is poorly conditioned if $k \geq 4$.

In the case that $k \geq p+1$, we find that $\mathcal{T}$ is locally well-conditioned provided that $\nu_{u_{0}}, \mu_{v_{0}}<2$, and is poorly conditioned if either $\nu_{u_{0}}$ or $\mu_{v_{0}}$ exceeds 2 . We note that $\nu_{u_{0}}>2$ if and only if

$$
(k+1)^{2}(4 p-5)+(k+1)\left(2 p^{2}-13 p\right)>8 p^{2} .
$$

If $p \geq 4$, then applying the fact that $k \geq p+1$, we find that $\nu_{u_{0}}>2$, so that $\mathcal{T}$ is poorly conditioned. If $p=3$, we find that $\mu_{v_{0}}>2$ if $k \geq 5$, while if $p=3$ and $k=4$ then $\nu_{u_{0}}, \mu_{v_{0}}<2$.

Now suppose that $p=2$. The conditions $\nu_{u_{0}}, \mu_{v_{0}}<2$ then reduce to

$$
3(k+1)^{2}-18(k+1)<32
$$


and

$$
4 k^{2}-15 k-57<0,
$$

respectively. It readily follows that $\mathcal{T}$ is locally well-conditioned if $k \leq 6$. On the other hand if $k \geq 7$, then $4 k^{2}-15 k-57>0$, which yields $\nu_{u_{0}}>2$, so that $\mathcal{T}$ is poorly conditioned.

Our final lemma discusses the class $T_{p}\left(l_{1,1}, \ldots, l_{n_{1}, 1} ; \ldots ; l_{1, k}, \ldots, l_{n_{k}, k}\right)$.

LEMMA 3.9. Suppose that $\mathcal{T}$ is a tree on $n$ vertices having two vertices a and $b$ with $\delta(a, b)=2$ and $d_{a}, d_{b} \geq 3$ and that the maximum distance between such pairs of vertices is 2 . Then $\mathcal{T}$ is poorly conditioned.

Proof. From the hypothesis, $\mathcal{T}$ is of the form $T_{p}\left(l_{1,1}, \ldots, l_{n_{1}, 1} ; \ldots ; l_{1, k}, \ldots, l_{n_{k}, k}\right)$ for some suitable lists of parameters. Note that by Corollary $3.2, \frac{m_{a, b}}{2(n-1)}+\frac{m_{b, a}}{2(n-1)}=2$. Suppose without loss of generality that $d_{b} \geq d_{a} \geq 3$. If $d_{b} m_{a, b} /(2 n-2) \geq 4$, then $\mathcal{T}$ is poorly conditioned. Suppose then that $d_{b} m_{a, b} /(2 n-2)<4$. Then necessarily $d_{a} m_{b, a} /(2 n-2)=d_{a}\left(2-m_{a, b} /(2 n-2)\right)>d_{a}\left(2-4 / d_{b}\right)=2 d_{a}-4\left(d_{a} / d_{b}\right)$. In particular, if $d_{a} \geq 4$, then $\mathcal{T}$ is poorly conditioned. Similarly, if $d_{a}=3$, we conclude that either $\mathcal{T}$ is poorly conditioned or that $4>6-\frac{12}{d_{b}}$, which yields $d_{b} \leq 5$.

Thus, it suffices to consider the case that apart from the central vertex $c_{1}$, there is at most one vertex of degree more than 3 , and that if such a vertex exists, its degree at most 5. Further, we may also assume that at most one vertex distinct from $c_{1}$ has degree at least 3 and is adjacent to a 2 -path, otherwise, by Lemma $3.4, \mathcal{T}$ is poorly conditioned.

In particular there is a vertex $a$ with $3 \leq d_{a} \leq 5$ which is adjacent to $c_{1}$ and to $d_{a}-1$ pendant vertices. Note that if $u$ is any vertex which is not one of the pendants adjacent to $a$, then there is a vertex $w$ adjacent to $u$ such that $m_{w, a} \geq$ $m_{v, a}=2 n-2 d_{a}-1$. Consequently, we see that $\mu_{a} \geq \frac{d_{a}}{2(n-1)}\left(1-\frac{d_{a}-1}{2 n-2}\right)\left(2 n-2 d_{a}-1\right)$. It is now straightforward to determine that if $n \geq 11$, that last expression exceeds 2 for each of $d_{a}=3,4,5$.

Now suppose that $n \leq 10$. In order to deal with the remaining cases, we give a slight refinement of the argument above. Let vertex $a$ be as above. Suppose that there are $p$ pendant vertices adjacent to $v$; note that necessarily $10 \geq n \geq d_{a}+p+4$, since $d_{b} \geq 3$. As above we have $m_{v, a}=2 n-2 d_{a}-1$, and note that each vertex which is distinct from $a$, not a pendant adjacent to $a$, and not a pendant adjacent to $v$, has the property that it is adjacent to a vertex $w$ such that $m_{w, a} \geq 2 n-2 d_{a}+2$. It now follows that

$$
\mu_{a} \geq \frac{d_{a}}{4(n-1)^{2}}\left(\left(2 n-2 d_{a}-1\right)\left(2 n-d_{a}-1\right)+3\left(2 n-2 d_{a}-1-p\right)\right) .
$$

Considering the cases $d_{a}=3,4,5$ in conjunction with the constraint that $10 \geq$ $n \geq d_{a}+p+4$, we find that the right side of (3.2) exceeds 2 (and so the corresponding tree is poorly conditioned) in all but two exceptional cases: $d_{a}=3, p=0, n=7$, and $d_{a}=4, p=0, n=8$. Those last two cases correspond to $T_{0}(1,1 ; 1,1)$ and $T_{0}(1,1,1 ; 1,1)$, respectively, and direct computations reveal that those trees are also poorly conditioned. 
The following characterization of locally well-conditioned trees is immediate from our discussion in this section.

THEOREM 3.10. Let $\mathcal{T}$ be a tree. Then $\mathcal{T}$ is locally well-conditioned if and only if it is isomorphic to one of the following forms:

i) A path on at most 5 vertices;

ii) $S\left(l_{1}, \ldots, l_{k}\right)$, where $l_{i} \leq 2, i=1, \ldots, k$;

iii) $R\left(l_{1}, \ldots, l_{k}, m_{1}, \ldots, m_{p}\right)$, where each $l_{i}$ and $m_{j} i s 1$, and where either $p=3$ and $3 \leq k \leq 4$, or $p=2$ and $2 \leq k \leq 6$.

\section{REFERENCES}

[1] A. Bondy and U. Murty. Graph Theory with Applications. Macmillan, London, 1977.

[2] S. Campbell and C. Meyer. Generalized Inverses of Linear Transformations. Dover, New York, 1991.

[3] G. Cho and C. Meyer. Comparison of perturbation bounds for the stationary distribution of a Markov chain. Linear Algebra and its Applications, 335:137-150, 2001.

[4] M. Haviv and L. Van der Heyden. Perturbation bounds for the stationary probabilities of a finite Markov chain. Advances in Applied Probability, 16:804-818, 1984.

[5] I. Ipsen and C. Meyer. Uniform stability of Markov chains. SIAM Journal on Matrix Analysis and Applications, 15:1061-1074, 1994.

[6] J. Kemeny and J. Snell. Finite Markov Chains. Van Nostrand, Princeton, 1960.

[7] S. Kirkland. The group inverse associated with an irreducible periodic nonnegative matrix. SIAM Journal on Matrix Analysis and Applications, 16:1127-1134, 1995.

[8] S. Kirkland. On a question concerning condition numbers for Markov chains. SIAM Journal on Matrix Analysis and Applications, 23:1109-1119, 2002.

[9] S. Kirkland and M. Neumann. Extremal first passage times for trees. Linear and Multilinear Algebra, 48:21-33, 2000.

[10] S. Kirkland, M. Neumann, and B. Shader. Applications of Paz's inequality to perturbation bounds for Markov chains. Linear Algebra and its Applications 268:183-196, 1998.

[11] E. Seneta. Non-negative Matrices and Markov Chains. Second edition, Springer-Verlag, New York, 1981. 